\title{
Hydromechanics of swimming propulsion. Part 2. Some optimum shape problems
}

\author{
By T. YAO-TSU WU \\ California Institute of Technology \\ Pasadena, California
}

(Received 21 July 1970)

The optimum shape problems considered in this part are for those profiles of a two-dimensional flexible plate in time-harmonic motion that will minimize the energy loss under the condition of fixed thrust and possibly also under other isoperimetric constraints. First, the optimum movement of a rigid plate is completely determined; it is necessary first to reduce the original singular quadratic form representing the energy loss to a regular one of a lower order, which is then tractable by usual variational methods. A favourable range of the reduced frequency is found in which the thrust contribution coming from the leading-edge suction is as small as possible under the prescribed conditions, outside of which this contribution becomes so large as to be hard to realize in practice without stalling. This optimum solution is compared with the recent theory of Lighthill (1970); these independently arrived-at conclusions are found to be virtually in agreement.

The present theory is further applied to predict the movement of a porpoise tail of large aspect-ratio and is found in satisfactory agreement with the experimental measurements. A qualitative discussion of the wing movement in flapping flight of birds is also given on the basis of optimum efficiency.

The optimum shape of a flexible plate is analysed for the most general case of infinite degrees of freedom. It is shown that the solution can be determined to a certain extent, but the exact shape is not always uniquely determinate.

\section{Introduction}

One of the most inspiring questions concerning the phenomena of aquatic animal propulsion and of flapping flights of birds and insects is invariably connected with the highest possible hydrodynamic efficiency. This problem has been brought up from time to time by various observers who have noted the impressive capabilitity of these animals in generating fast movements at low energy cost. According to the first principle of energy balance or momentum consideration, as has been explained in part 1 of this paper (Wu 1971), much can already be said about the desirable shapes of body movement: that at large Reynolds numbers, a thin two-dimensional plate gains thrust by sending a transverse wave from head to tail, with amplitude slightly increasing towards the rear, thereby achieving a forward swimming velocity somewhat less than the phase velocity of the body wave form. As for the tail of large aspect-ratio 
of some high-performance fish, the tail should move nearly tangentially to the path traversed in the space by the body wave form. These basic features have been elegantly elucidated, with perhaps more physical reasoning, in an excellent review by Lighthill (1969). However, it would still be of great interest to resolve a quantitative determination of the optimum shape under some appropriate constraints.

The problem of the optimum shape is interesting in its own right from the mathematical point of view, since the effective methods of solution do not seem to fall into the known categories of the calculus of variation. The special case of a two-dimensional waving plate in harmonic motion has been treated by Wang (1966), who adopted a discretized Fourier representation of the body motion, and found that his solutions exist only for a set of eigenvalues. However, it is found in the present study that this optimum shape problem is basically not an eigenvalue problem, and therefore merits a new discussion. On physical grounds, it would be indeed difficult to see the significance of the idea that the shape function can have eigensolutions.

In this part we shall consider the optimum shape problem only for the case of two-dimensional flexible plate, of negligible thickness, in harmonic motion. (Some three-dimensional problems will be treated in part 3 of this paper.) The two-dimensional theory is reckoned to have utility in problems of lifting surfaces of large aspect-ratio, such as the tails of some cetaceans and highperformance game fish (the lunate tails: swordfish, tuna, albacore, porpoises, etc.), and even the wings of most birds and some insects. The optimum shape problem is concerned with those profiles or movements that will minimize the energy loss under the condition of fixed thrust (required to overcome the viscous drag), and possibly also under other isoperimetric constraints. First, the optimum movement of a rigid plate is determined by reducing the original singular problem to a regular one of a lower rank. This optimum solution is found to depend on two variables: one being the reduced frequency and the other a 'proportional-loading parameter', defined as the prescribed thrust coefficient divided by the dimensionless heaving amplitude squared. For given loading parameter, a favourable range of the reduced frequency is found in which the thrust contribution coming from the leading edge suction is as small as possible under the prescribed conditions. This consideration seems to provide the optimum range of the reduced frequency utilized in practice.

These theoretical results are further applied to predict the movement of a porpoise tail, and comparisons made with the experimental investigation of Lang \& Daybell (1963). As a related problem of interest, the optimum movement of a flapping wing of some birds or flatfish is discussed qualitatively.

The general problem of optimum shape of a flexible surface having an infinite degree of freedom is finally analysed and discussed. It is found that the solution can be determined to a certain extent, and, with the additional degrees of freedom, the optimum efficiency can be further improved from the rigid-plate value, but the exact shape is not uniquely determinate. 


\section{Statement of the optimum shape problem}

As a starting point, the following basic results are reproduced from part 1 (Wu 1971). The class of motion treated here is that of a two-dimensional flexible plate, immersed parallel to a uniform stream of velocity $U$ in the $x$-direction, and performing a harmonic transverse motion

$$
y=h(x, t)=h_{1}(x) \exp (j \omega t) \quad(-1 \leqslant x \leqslant 1),
$$

which is assumed to be continuous in $-1 \leqslant x \leqslant 1$ and to possess a Fourier expansion

with $\quad \beta_{n}=\frac{2}{\pi} \int_{0}^{\pi} h(x, t) \cos n \theta d \theta \quad(n=0,1 \ldots N \leqslant \infty)$.

$$
h(x, t)=\frac{1}{2} \beta_{0}+\sum_{n=1}^{N} \beta_{n} \cos n \theta \quad(x=\cos \theta, 0 \leqslant \theta \leqslant \pi),
$$

This motion generates at the plate a transverse flow velocity,

$$
V(x, t)=\frac{\partial h}{\partial t}+U \frac{\partial h}{\partial x}=U\left(\frac{\partial h}{\partial x}+j \sigma h\right) \quad(\sigma=\omega / U),
$$

which can also be expanded in a Fourier series,

with

$$
V(x, t)=\frac{1}{2} b_{0}+\sum_{n=1}^{N} b_{n} \cos n \theta \quad(x=\cos \theta),
$$

In (2)-(4) and in the sequel, the harmonic time factor $\exp (j \omega t)$ of $h, V, \beta_{n}$ 's and $b_{n}$ 's is always taken as understood. The reduced frequency $\sigma$ is referred to halfchord $l$, which is being taken as the unit length, or

$$
\sigma=\omega l / U
$$

The time averages of thrust $\bar{T}$, energy loss $\bar{E}$, and power required $\bar{P}$ can be put in the coefficient form,

$$
\begin{aligned}
& C_{E} \equiv \bar{E} /\left(\frac{1}{4} \pi \rho U^{3} l\right)=B(\sigma)\left(b_{0}+b_{1}\right)\left(b_{0}^{*}+b_{1}^{*}\right) / U^{2} \\
& C_{P} \equiv \bar{P} /\left(\frac{1}{4} \pi \rho U^{3} l\right)=\operatorname{Re}\left\{-(j \sigma / U)\left(b_{0}+b_{1}\right)\left[\left(\beta_{0}^{*}-\beta_{1}^{*}\right) \Theta(\sigma)+\beta_{1}^{*}\right]\right\} \\
& C_{T^{\prime}} \equiv \bar{T} /\left(\frac{1}{4} \pi \rho U^{2} l\right)=C_{P}-C_{E}
\end{aligned}
$$

where the symbols with $*$ stand for their complex conjugates, $\Theta(\sigma)$ denotes Theodorsen's function

$$
\begin{gathered}
\Theta(\sigma)=K_{1}(j \sigma) /\left[K_{0}(j \sigma)+K_{1}(j \sigma)\right]=\mathscr{F}+j \mathscr{G}, \\
B(\sigma)=\mathscr{F}-\left(\mathscr{F}^{2}+\mathscr{G}^{2}\right),
\end{gathered}
$$

$K_{n}$ being the modified Bessel function of the second kind, $\mathscr{F}$ and $\mathscr{G}$ being the real and imaginary parts of $\Theta$.

The general optimum shape problem can be stated as follows: Within the class of shape function $h$ as specified above, find the optimum one which will minimize $C_{E}$ under the condition of fixed thrust coefficient, say

$$
C_{T}=C_{T, 0}>0 \text {, }
$$

the reduced frequency being regarded as a fixed parameter. 
As has been pointed out in part $1, C_{E} \geqslant 0$ for $\sigma>0$ with any admissible $h$, consequently the extremal solution of $C_{E}$ will not be negative. Here, $C_{T}$ rather than $C_{p}$ is chosen to be a fixed positive quantity for the sole reason that the result will always give a meaningful hydrodynamic efficiency:

$$
\eta=C_{T} / C_{p}=C_{T, 0} / C_{p}=C_{T, 0} /\left(C_{T, 0}+C_{E}\right)
$$

If $C_{p}$ is fixed instead, the solution of $C_{T}$ may become negative. Aside from this point, there is no fundamental difference whether $C_{T}$ or $C_{p}$ is fixed.

It is of interest to note that only the first two Fourier coefficients of $h$ and of $V$ appear in the expressions for $C_{E}, C_{P}$ and $C_{T}$. Since $h$ and $V$ are related by a differential equation (3), $b_{n}$ can be expressed in terms of $\beta_{n}$ 's upon substituting (2), (4) in (3). Conversely, if $V$ is first prescribed by a set of $b_{n}$ 's, $\beta_{n}$ can be evaluated in terms of $b_{n}$ upon integration of (3), thus introducing a complementary solution proportional to $\exp (-j \sigma x)$. In either case, $b_{0}$ and $b_{1}$ will depend on all the $\beta_{n}$ 's which are admitted to $h$, or $\beta_{0}$ and $\beta_{1}$ will depend on all. the $b_{n}$ 's admitted to $V$. If the number $N$ of the terms in (2), (4), is taken to be infinite, so that $h$ and $V$ each will define a vector space of infinite dimensions, the problem can be recast somewhat as follows. Define the scalar product of two functions $h(x)$ and $g(x)$ over $-1 \leqslant x \leqslant 1$ by

$$
(h, g)=\frac{2}{\pi} \int_{0}^{\pi} h(x) g^{*}(x) d \theta \quad(x=\cos \theta),
$$

then (6), (7) may be written

$$
\begin{aligned}
& C_{E}=U^{-2} B(\sigma)(V, 1+x)\left(V^{*}, 1+x\right), \\
& C_{P}=\operatorname{Re}\left\{(-j \sigma / U)(V, 1+x)\left[\left(h^{*}, 1\right) \Theta(\sigma)+\left(h^{*}, x\right)(1-\Theta(\sigma))\right]\right\} .
\end{aligned}
$$

A striking feature here is that there are only three different scalar products involving $h$ and $V$ that can be subjected to variation, from which the optimum $h$ is to be determined.

The number of the Fourier coefficients, or equivalently, the number of scalar products can be increased by a few if the waving plate propels itself without external agencies whilst the recoil conditions are imposed on the plate's being free from lateral and angular recoil. These recoil conditions require that the hydrodynamic lift $L$ and moment $M$ must be equal and opposite to the time-rate of change of the lateral and angular momentum of the body (see (40), (41), (56) of part 1), or

$$
\begin{aligned}
\left(b_{0}+b_{1}\right) \Theta(\sigma)+\frac{1}{2} j \sigma\left(b_{0}-b_{2}\right) & =+\frac{U \sigma^{2}}{\pi \rho} \int_{-1}^{1} m(x) h(x) d x \\
\left(b_{0}+b_{1}\right) \Theta(\sigma)-\left(b_{1}+b_{2}\right)-\frac{1}{4} j \sigma\left(b_{1}-b_{3}\right) & =-\frac{2 U \sigma^{2}}{\pi \rho} \int_{-1}^{1} x m(x) h(x) d x
\end{aligned}
$$

where $m(x)$ is the plate mass per unit distance in $x$. Conditions (15), (16) are additional isoperimetric conditions to be satisfied together with (10) in extremizing $C_{E}$. These two conditions can, however, be disregarded or accounted for separately, when this two-dimensional theory is applied to evaluate the propulsion of a lifting surface (of large aspect-ratio) which is only a part of the self- 
propelling body, such as the tail of certain cetaceans and Lunate tails, or the wings of birds, since the question of recoil requires a consideration of the entire body.

\section{Optimum movement of a rigid-plate wing}

The basic nature of this optimum shape problem can be best seen from the following simple case:

so that

$$
\begin{gathered}
h(x, t)=\left[\frac{1}{2} \xi_{0}+\left(\xi_{1}+j \xi_{2}\right) x\right] \exp (j \omega t) \quad(|x| \leqslant 1), \\
\beta_{0}=\xi_{0} \exp (j \omega t), \quad \beta_{1}=\left(\xi_{1}+j \xi_{2}\right) \exp (j \omega t),
\end{gathered}
$$

where $\xi_{0}, \xi_{1}, \xi_{2}$ are real. The above $h$ represents a rigid plate performing a heaving with amplitude $\frac{1}{2} \xi_{0}$ and a pitching about $x=0$ with amplitude $\left|\xi_{1}+j \xi_{2}\right|$ at a phase angle $\tan ^{-1}\left(\xi_{2} / \xi_{1}\right)$ leading the heaving motion. This special case, though about the simplest in form for the general optimum problem, still embraces a considerable interest for its result may cast light on the tail motion of some high-performance fish and cetacean, as well as on the flapping wings of birds in flight.

Before we proceed further, we list here two fundamental cases:

(i) Heaving only, so that $\xi_{1}=\xi_{2}=0$ and only $\xi_{0} \neq 0$. Then, by (2)-(4),

$$
b_{0}=U j \sigma \xi_{0} \exp (j \omega t), \quad b_{1}=0 .
$$

The corresponding $C_{T}, C_{E}, C_{P}$ are

$$
C_{E}=\sigma^{2} B(\sigma) \xi_{0}^{2}, \quad C_{P}=\sigma^{2} \mathscr{F}(\sigma) \xi_{0}^{2}, \quad C_{T}=\sigma^{2}\left(\mathscr{F}^{2}+\mathscr{G}^{2}\right) \xi_{0}^{2} .
$$

The hydrodynamic efficiency of heaving propulsion,

$$
\eta_{\text {heav }}(\sigma)=C_{T} / C_{P}=\left(\mathscr{F}^{2}+\mathscr{G}^{2}\right) / \mathscr{F},
$$

is seen to depend on $\sigma$ only, decreasing monotonically from $\eta_{h}(0)=1$ to $\eta_{h}(\infty)=0 \cdot 5$, as shown in figure 1 . This general trend of $\eta_{h}$ is readily verified from the known asymptotic behaviour of $\mathscr{F}$ and $\mathscr{G}$ that, for $\sigma \ll 1$,

$$
\begin{gathered}
\mathscr{F}(\sigma) \sim 1-\frac{\pi \sigma}{2}-\sigma^{2}\left(\log ^{2} \frac{2}{\gamma_{1} \sigma}-\frac{\pi^{2}}{4}\right)+O\left(\sigma^{3} \log ^{2} \sigma\right) \quad\left(\gamma_{1}=1 \cdot 781 \ldots\right) \\
\mathscr{G}(\sigma) \sim-\sigma(1-\pi \sigma) \log \frac{2}{\gamma_{1} \sigma}+O\left(\sigma^{3} \log ^{3} \sigma\right)
\end{gathered}
$$

and, for $\sigma \gg 1$,

$$
\mathscr{F}(\sigma) \sim \frac{1}{2}\left[1+\frac{1}{8 \sigma^{2}}+O\left(\sigma^{-4}\right)\right], \quad \mathscr{G}(\sigma) \sim-\frac{1}{8 \sigma}\left[1-\frac{11}{128 \sigma^{2}}+O\left(\sigma^{-4}\right)\right] .
$$

(ii) Pitching only, so that $\xi_{0}=0$ and we may also set $\xi_{2}=0$ as a reference phase,

$$
\beta_{0}=0, \quad \beta_{1}=\xi_{1}, \quad b_{0}=2 U \xi_{1}, \quad b_{1}=U j \sigma \xi_{1},
$$

in which the harmonic time factors are omitted as understood. Whence, from $(6)-(8)$,

where

$$
C_{E}=B(\sigma) Q_{22} \xi_{1}^{2}, \quad C_{P}=\sigma P_{22} \xi_{1}^{2}, \quad C_{T}=T_{22} \xi_{1}^{2}
$$


In this pure-pitching mode, both $C_{E}$ and $C_{P}$ are positive definite for $\sigma>0$. But $T_{22}(\sigma)=0$ has one real root,

$$
T_{22}\left(\sigma_{0}\right)=0 \text { for } \sigma_{0}=1 \cdot 781,
$$

and $T_{22} \lesseqgtr 0$ according as $\sigma \lesseqgtr \sigma_{0}$ (see figure 2 for its numerical value). When $C_{T}>0$, we may define the hydrodynamic efficiency

$$
\eta_{\mathrm{p} 1 \mathrm{tch}}(\sigma)=\frac{C_{T}}{C_{P}}=\frac{T_{22}(\sigma)}{\sigma P_{22}(\sigma)} \quad\left(\sigma>\sigma_{0}\right)
$$

which is found to increase monotonically from $\eta_{p}\left(\sigma_{0}\right)=0$ to $\eta_{p}(\infty)=0.5$, as shown in figure 1. We further note that in either case (i) or (ii), power must be

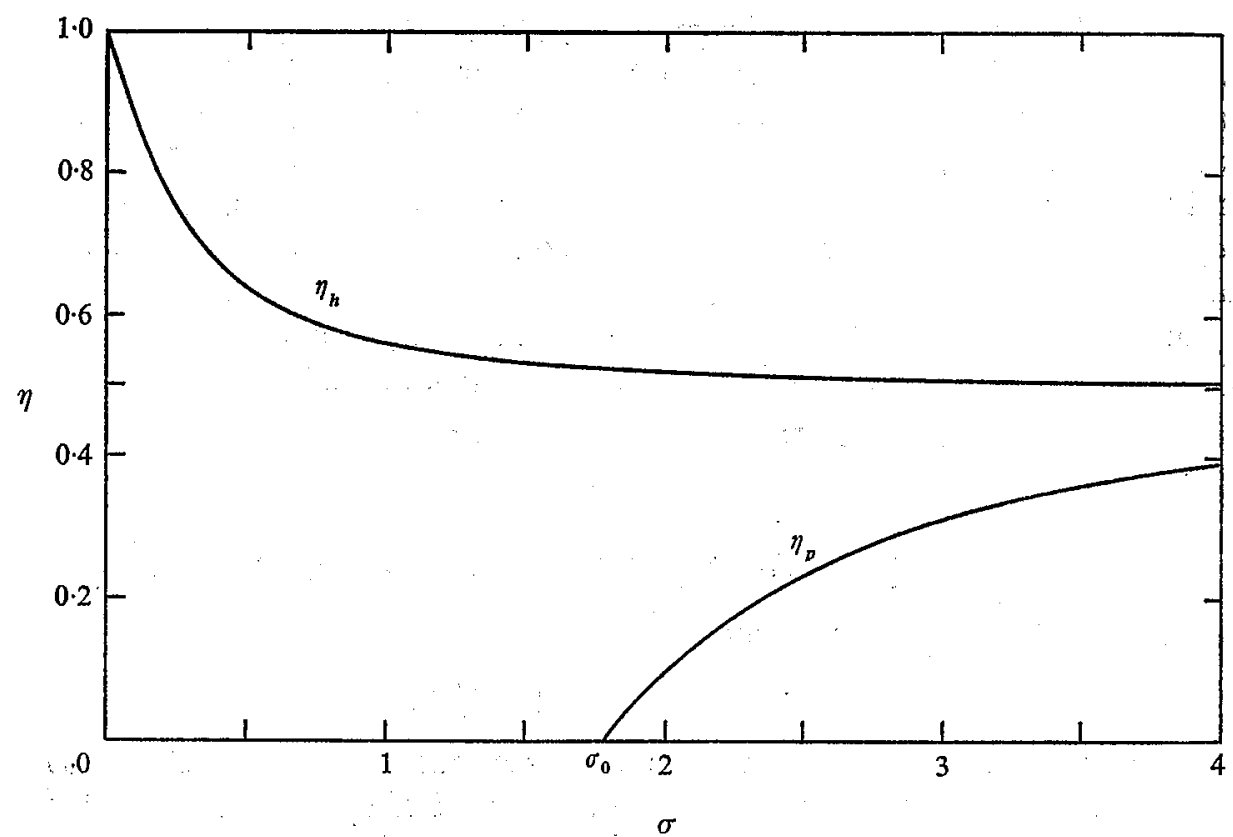

Frgure 1. Hydrodynamic efficiency of heaving propulsion, $\eta_{h}(\sigma)$, and of pitching propulsion, $\eta_{p}(\sigma)$, the latter being defined for the reduced frequency $\sigma>\sigma_{0}=\mathrm{I} \cdot 78 \mathrm{I}$.

supplied to maintain the motion, consequently it is impossible to extract energy from the fluid in so far as either (i) or (ii) is concerned separately. The main objective of optimization is then to determine if the efficiency can be greatly improved when both heaving and pitching modes are admitted.

Returning to the combined motion, the value of $\left(b_{0}+b_{1}\right)$ corresponding to $h$ of (17) is, by (3) and (4),

$$
b_{0}+b_{1}=U \exp (j \omega t)\left[j \sigma \xi_{0}+(2+j \sigma)\left(\xi_{1}+j \xi_{2}\right)\right] .
$$

We note here that $b_{0}+b_{1}=0$ when

$$
\xi_{1}=\xi_{1} \equiv-\sigma^{2}\left(\sigma^{2}+4\right)^{-1} \xi_{0}, \quad \xi_{2}=\xi_{2} \equiv-2 \sigma\left(\sigma^{2}+4\right)^{-1} \xi_{0} .
$$

At the same time, $C_{E}, C_{P}$ and $C_{T}$ all vanish with $\left(b_{0}+b_{1}\right)$, according to (6)-(8). This particular set of values $\left(\xi_{0}, \xi_{1}, \xi_{2}\right)$ will be seen to play a significant role in the 
where $\mu$ is related to the old multiplier $\lambda^{\prime}$ (the actual relationship being immaterial). The first two eigenvalues $\mu_{1}, \mu_{2}$, being a double root of zero, yield the same eigenvector $\left(\xi_{0}, \xi_{1}, \xi_{2}\right)$ with $\xi_{1}, \hat{\xi}_{2}$ given by $(29)$. But, as noted before, $b_{0}+b_{1}=0$ when $\xi_{1}=\xi_{1}, \xi_{2}=\hat{\xi}_{2}$, thereby making $C_{E}, C_{P}$ and $C_{T}$ all vanish. Clearly this eigenvector is not the solution since condition (10) can not be satisfied. (The generalized eigenvector of rank 2 in the sense $\mathbf{Q}^{2} \boldsymbol{\xi}=0, \mathbf{Q} \xi \neq 0$ for the multiple root $\mu_{2}$ does not exist, nor does any generalized eigenvector of higher ranks.) The third eigenvalue $\mu_{3}$ gives the eigenvector having $\xi_{0}=0$; the resulting $C_{E}$ therefore becomes proportional to $C_{T}$, implying that this last eigenvector is a stationary solution. This also shows that the foregoing method based on the spectral theory, as was used by Wang (1966), does not work.

The correct approach is found by noting that since $\mathbf{Q}$ is singular, but nonsingular in the second order, the quadratic form $C_{E}$ can always be reduced to a non-singular form in two variables. In fact, in terms of the new variables,

$$
\zeta_{0}=\xi_{0} /\left(4+\sigma^{2}\right), \quad \zeta_{1}=\xi_{1}-\xi_{1}, \quad \zeta_{2}=\xi_{2}-\xi_{2}
$$

with $\hat{\xi}_{1}, \hat{\xi}_{2}$ given by (29), $C_{E}$ and $C_{P}$ in (30) reduce to

$$
\begin{aligned}
& C_{E}=B(\sigma) Q_{22}\left(\zeta_{1}^{2}+\zeta_{2}^{2}\right), \\
& C_{P}=\sigma\left\{P_{22}\left(\zeta_{1}^{2}+\zeta_{2}^{2}\right)+2 A_{1} \zeta_{0} \zeta_{1}+2 A_{2} \zeta_{0} \zeta_{2}\right\} \\
& A_{1}=P_{12} Q_{22}-Q_{12} P_{22}, \quad A_{2}=P_{13} Q_{33}-Q_{13} P_{33}
\end{aligned}
$$

Now it is clear that, while $C_{P}$ spans the whole vector space $\left(\zeta_{0}, \zeta_{1}, \zeta_{2}\right), C_{E}$ spans only its subspace $\left(\zeta_{1}, \zeta_{2}\right)$. Obviously the surface $C_{E}=$ const. $=C_{E, 0}>0$ is a circular cylinder with its central axis along the $\zeta_{0}$-axis. The quadric $C_{P}=$ const. $=C_{P, 0}>0$ is seen to be an oblique hyperboloid of one sheet, since its intersection with the plane $\zeta_{0}=$ const. is a circle centred at $\left(-A_{1} \zeta_{0} / P_{22},-A_{2} \zeta_{0} / P_{22}\right)$, of radius $\left[\left(A_{1}^{2}+A_{2}^{2}\right)\left(\zeta_{0} / P_{22}\right)^{2}+C_{P} /\left(\sigma P_{22}\right)\right]^{\frac{1}{2}}$. The extremal solutions under condition (10) are therefore given by the points in the subspace $\left(\zeta_{1}, \zeta_{2}\right)$ at which $\left(\operatorname{grad} C_{E}\right)$ is proportional to (grad $C_{P}$ ). This situation is depicted in figure 3 in terms of $C_{E}$ and $C_{T}$. Thus, after setting the derivatives of $C_{E}^{\prime}=\left(C_{E}-\lambda^{\prime \prime} C_{P}\right)$ with respect to $\zeta_{1}$ and $\zeta_{2}$ to zero, we obtain

$$
\zeta_{1}=\lambda A_{1} \zeta_{0}, \zeta_{2}=\lambda A_{2} \zeta_{0}
$$

where $\lambda$ is a Lagrange multiplier. Upon substituting (38) in (35)-(36),

$$
\begin{aligned}
& C_{E}=B Q_{22} \lambda^{2}\left(A \zeta_{0}\right)^{2}, \quad A^{2}=A_{1}^{2}+A_{2}^{2}, \\
& C_{P}=\sigma\left(P_{22} \lambda^{2}+2 \lambda\right)\left(A \zeta_{0}\right)^{2} .
\end{aligned}
$$

Now, application of condition (10), or $C_{P}-C_{E}=C_{T, 0}$, results in a quadratic equation for $\lambda$,

where

$$
\begin{gathered}
T_{22}(\sigma) \lambda^{2}+2 \sigma \lambda=\bar{C}_{T, 0}\left(4+\sigma^{2}\right)^{2} / A^{2}, \\
\bar{C}_{T, 0}=C_{T, 0} / \xi_{0}^{2}, \quad A^{2}=A_{1}^{2}+A_{2}^{2},
\end{gathered}
$$

and $T_{22}(\sigma)$ is given by (25). $\vec{C}_{T, 0}$ will be called the 'proportional-loading parameter'. The multiplier $\lambda$ therefore has two solutions

$$
\left.\begin{array}{l}
\lambda_{1} \\
\lambda_{2}
\end{array}\right\}=\frac{\sigma}{T_{22}}\left\{-1 \pm(1+\Lambda)^{\frac{1}{2}}\right\}, \quad \Lambda=\bar{C}_{T, 0} T_{22}\left(\frac{\sigma^{2}+4}{\sigma A}\right)^{2}
$$


$\lambda_{1}, \lambda_{2}$ depend on two parameters: $\sigma$ and $\bar{C}_{T, 0}$. By virtue of the behaviour $T_{22}(\sigma) \gtrless 0$ according as $\sigma \gtrless \sigma_{0}=1 \cdot 781$, it follows that for fixed $\bar{C}_{T, 0}>0$, $\Lambda\left(\sigma, \bar{C}_{T, 0}\right)$ increases monotonically from $-\infty$ to 0 as $\sigma$ moves from $\sigma=0$ to $\sigma_{0}$. . Consequently, $\lambda_{1}, \lambda_{2}$ will be real (as are required to be physically meaningful) if $\Lambda \geqslant-1$; or, equivalently, for

$$
\sigma \geqslant \sigma_{c}\left(\bar{C}_{T, 0}\right), \text { where } \Lambda\left(\sigma_{c}, \bar{C}_{T, 0}\right)=-1 .
$$

The solution $\sigma_{c}=\sigma_{c}\left(\bar{C}_{T, 0}\right)$ is shown in figure 4 to lie between $\sigma_{c}(0)=0$ and $\sigma_{c}(\infty)=\sigma_{0}$. For given $\bar{C}_{T, 0}>0$, the real optimum solution therefore exists only

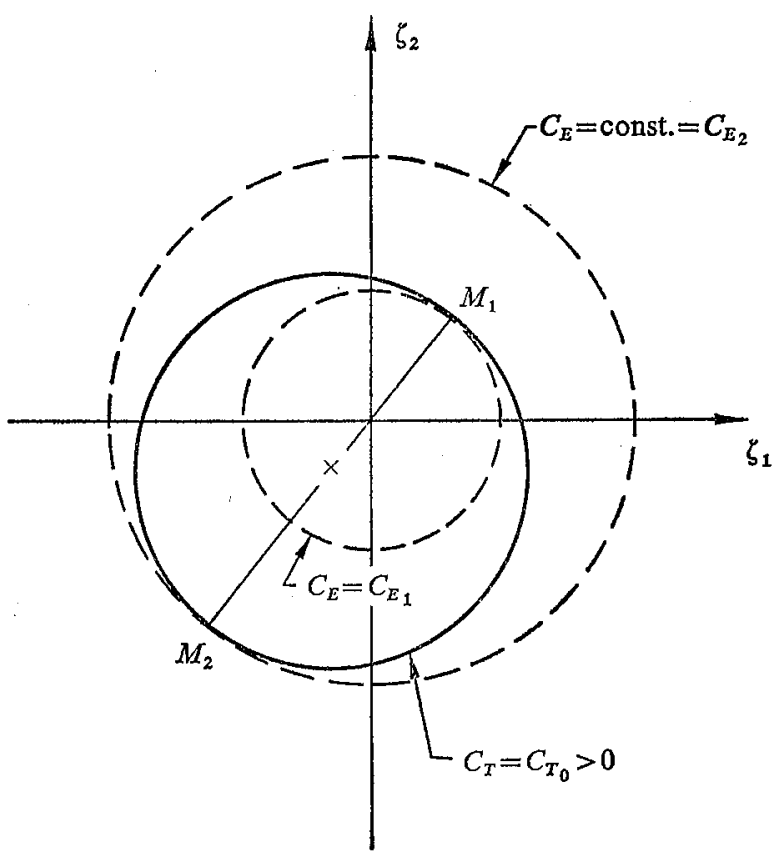

FiquRe 3. For $\zeta_{0}$ fixed, the quadric $C_{T}=C_{T, 0}>0$ is an off-centred circle which meets tangentially the surface $C_{E}=C_{E, 1}$ at $M_{1}$ and $C_{E}=C_{E, 2}$ (> $C_{E, 1}$ ) at $M_{2}$. The points $M_{1}$ and $M_{2}$ correspond, respectively, the maximum and minimum hydrodynamic efficiency under condition of fixed $C_{T}=C_{T, 0}$.

for $\sigma \geqslant \sigma_{e}$. Within this range, $\lambda_{1}$ is positive, numerically smaller than $\lambda_{2}$, and corresponds to the highest efficiency attainable under condition (10),

$$
\eta_{\max }=\frac{C_{T, 0}}{C_{P}}=1-\frac{C_{z}}{C_{P}}=1-\frac{B Q_{22} \lambda_{1}}{\sigma\left(P_{22} \lambda_{1}+2\right)} \quad\left(\sigma \geqslant \sigma_{c}\right) .
$$

The lowest efficiency $\eta_{\mathrm{min}}$ that can be realized under the same conditions (10) is given by the last expression of (44) with $\lambda_{1}$ replaced by $\lambda_{2}$. For any combination of $\zeta_{0}, \zeta_{1}, \zeta_{2}$ different from (38), the efficiency $\eta$ is $\eta_{\min }<\eta<\eta_{\max }$ so long as $\bar{C}_{T, 0}$ is kept fixed.

The following salient features of the solution are noteworthy.

(i) At $\sigma=\sigma_{c}, \Lambda=-1$, so that $\lambda_{1}=\lambda_{2}=-\sigma_{e} / T_{22}\left(\sigma_{e}\right)$; hence, from (44) and (25), it follows that for arbitrary $\vec{C}_{T, 0}>0$

$$
\eta_{\max }\left(\sigma_{c}\right)=\left[2+P_{22}\left(\sigma_{c}\right) \lambda_{1}\left(\sigma_{c}\right)\right]^{-1}<\frac{1}{2}
$$


in virtue of $P_{22}(\sigma)>0$ and $\lambda_{1}\left(\sigma_{c}\right)>0$. This shows that, in the frequency range near $\sigma_{c}, \eta_{\max }$ cannot be very impressive. In fact, $\eta_{\max }\left(\sigma_{c}\right) \rightarrow 0$ monotonically as $\bar{C}_{T, 0} \rightarrow \infty$, since in this limit $T_{22}\left(\sigma_{c}\right) \rightarrow T_{22}\left(\sigma_{0}\right)=0$, and hence $\lambda_{1} \rightarrow \infty$ (see figure 4).

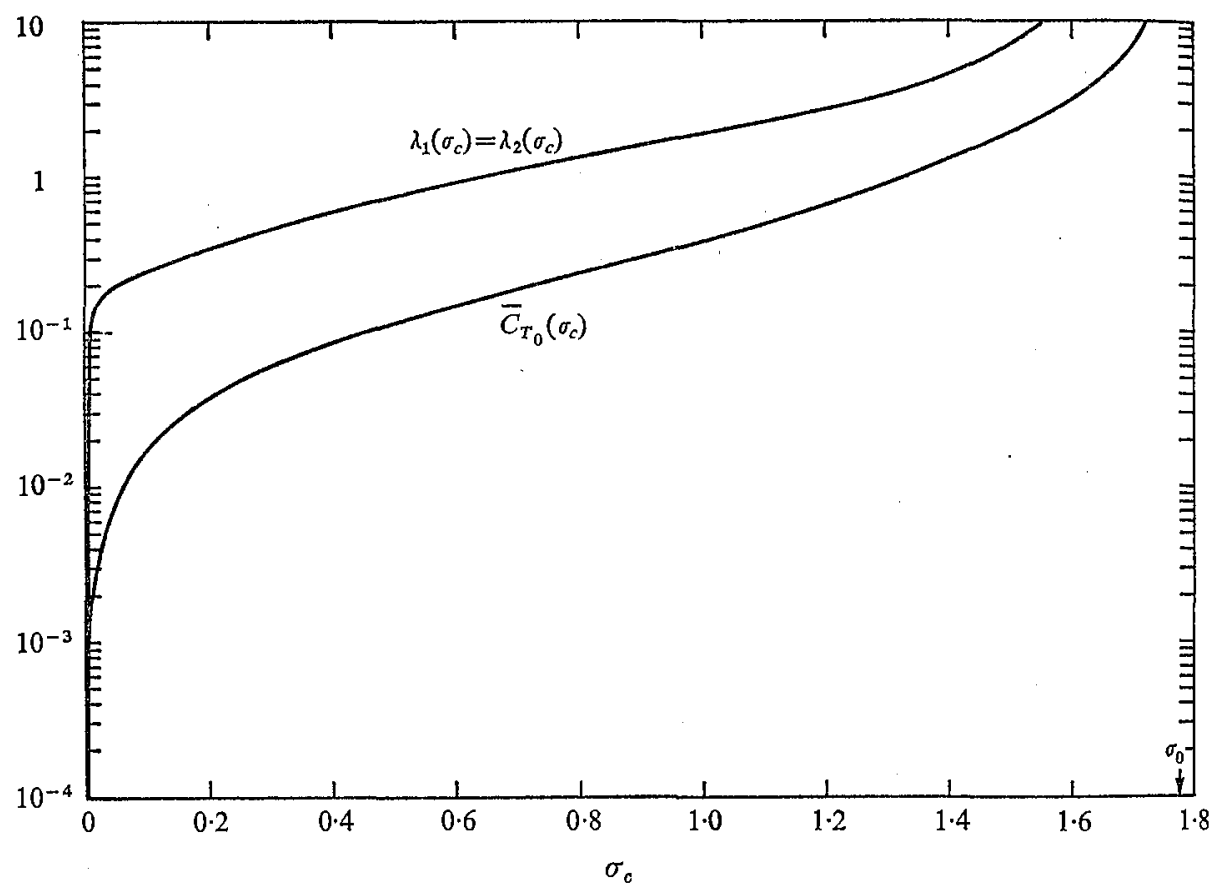

FIguRe 4. For given $\bar{C}_{T, 0}>0$, the critical $\sigma_{c}$ marks the lower bound of the reduced frequency $\sigma$, below which no real optimum solution exists.

However, when $\bar{C}_{T, 0} \ll 1, \sigma_{c}$ is also small. By making use of the asymptotic expansions (21) for $\mathscr{F}$ and $\mathscr{G}$, we readily deduce that

$$
\begin{gathered}
\sigma_{c} \sim 2 \pi \bar{C}_{T, 0}\left\{1+\bar{C}_{T, 0}\left[\left(2-\frac{1}{\pi}\right) \log \frac{2}{\gamma_{1} \bar{C}_{T, 0}}+\frac{\pi}{2}\right]+O\left(\sigma_{c}^{2} \log ^{2} \sigma_{c}\right)\right\}, \\
\eta_{\max } \sim \frac{1}{2}\left\{1-\frac{\sigma}{2 \pi} \log \frac{2}{\gamma_{1} \sigma}+\left(\frac{\sigma-\sigma_{c}}{\sigma}\right)^{\frac{1}{2}}\left[1-\frac{\pi+4}{4} \sigma \log \frac{2}{\gamma_{1} \sigma}\right]\right\},
\end{gathered}
$$

the last expression being valid for $0 \leqslant\left(\sigma-\sigma_{c}\right) \ll 1$. Note that $d \eta\left(\sigma_{c}\right) / d \sigma=\infty$; thus $\eta_{\max }$ rises rapidly from $\eta_{\max }\left(\sigma_{c}\right)$ as $\sigma$ increases from $\sigma_{c}$.

(ii) Near $\sigma=\sigma_{0}\left(\sigma_{0}\right.$ defined by (26)), we deduce from (26), (44), and (22) that

$$
\lambda_{1}(\sigma)=\frac{1}{2} \bar{C}_{T, 0}\left(\sigma_{0}^{2}+4\right)^{2} /\left(\sigma_{0} A^{2}\right)+O\left(\left|\sigma-\sigma_{0}\right|\right)
$$

$\eta_{\max }(\sigma)=\left[1+\frac{1}{2} P_{22}\left(\sigma_{0}\right) \lambda_{1}\left(\sigma_{0}\right)\right]^{-1}+O\left(\left|\sigma-\sigma_{0}\right|\right) \simeq\left[1+\frac{8}{9} \bar{C}_{x, 0}\right]^{-1}+O\left(\left|\sigma-\sigma_{0}\right|\right)$.

We note that $\eta_{\max }\left(\sigma_{0}\right)$ is already more than twice $\eta_{\max }\left(\sigma_{c}\right)$.

(iii) For $\bar{C}_{T, 0} \ll 1$ and $\sigma_{c} \ll \sigma, \Lambda$ is small, say $\Lambda=\epsilon \ll 1$. Then $\lambda_{1}=O(\epsilon)$, consequently $\eta_{\max }=1-O(\epsilon)$ for $\sigma_{c} \ll \sigma$, indicating that high efficiencies can be achieved in the neighbourhood of $\bar{C}_{T, 0}=C_{T, 0} / \xi_{0}^{2}=0$. In this operating region the amplitude of heaving of course must not vanish $\left(\xi_{0} \neq 0\right)$. In the limit as $\bar{C}_{T, 0} \rightarrow 0$, $\lambda_{1} \rightarrow 0$, and hence $\zeta_{1}, \zeta_{2} \bar{T}, \bar{E}, \bar{P}$ all tend to zero whereas $\eta_{\max } \rightarrow 1$, corresponding to the singular case already mentioned in part 1. 
These results are shown in figures 6 and 7 for several values of $\bar{C}_{T, 0}$. For $\sigma$ sufficiently large, say $\sigma>2$, curves of different $\bar{C}_{T, 0}(<1)$ approach to a common asymptotic representation:

$$
Z_{p} \sim \frac{\sigma^{2}+2\left(1-5 \bar{C}_{T, 0} / 3\right)}{\sigma^{2}+4}, \quad \alpha_{p} \sim \pi+\tan ^{-1}\left[\frac{2}{\sigma}\left(1+\frac{2}{3} \bar{C}_{T, 0}\right)\right] .
$$

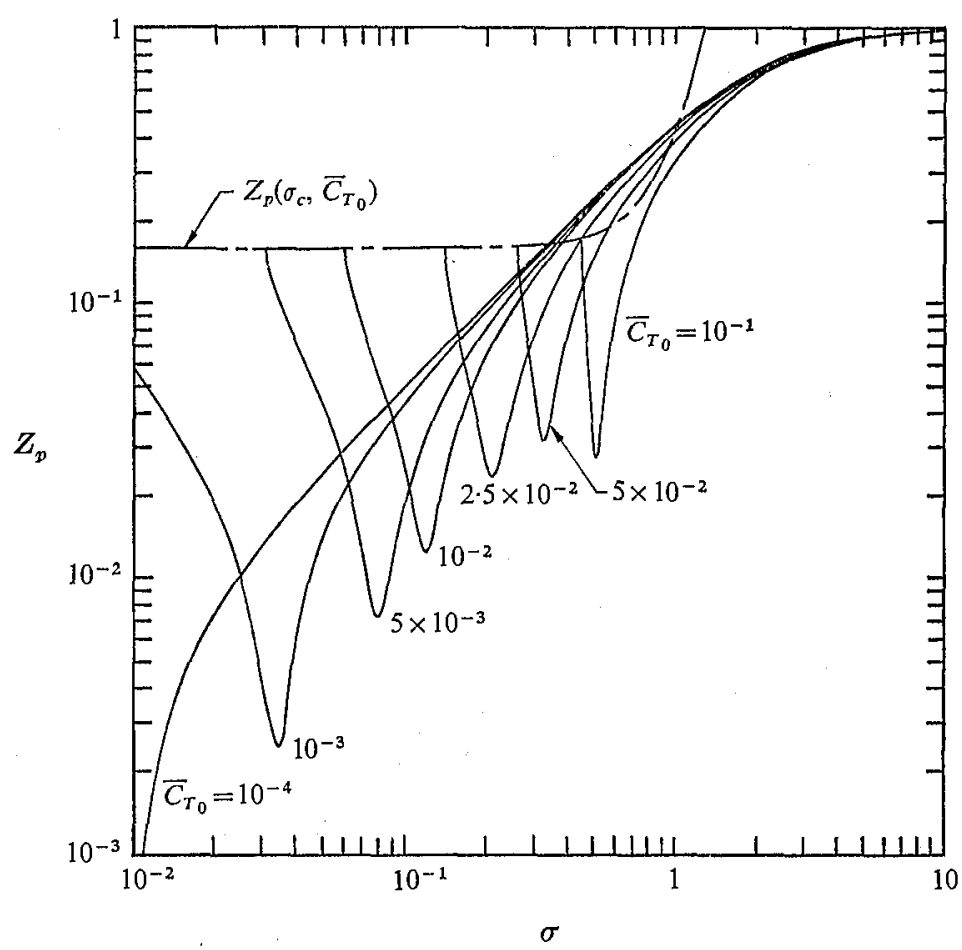

FIGURE 6. The amplitude ratio (pitching/heaving) $Z_{p}\left(\sigma, \bar{C}_{T, 0}\right)$.

In summary, we first notice the advantage of operating at small values of $\bar{C}_{T, 0}$, corresponding to sufficiently large heaving amplitude. A smaller $\bar{C}_{T, 0}$ renders the optimum solution valid to lower frequencies $\sigma$, and makes $\eta_{\max }$ greater at the same $\sigma$. As $\sigma$ increases from $\sigma_{c}$, the pitching-heaving amplitude ratio $Z_{p}$ first decreases to a minimum, then increases steadily to a common asymptote. Over the same range of $\sigma$, the phase difference $\alpha_{p}$ changes very rapidly at first, followed by a much slower variation at higher $\sigma$. A rather sophisticated control would therefore be necessary if the operating range of $\sigma$ is chosen in which fast variations of $Z_{p}$ and $\alpha_{p}$ may take place. It is a remarkable result that in the higher range of $\sigma$, very high efficiencies can be realized with an appropriate interplay between the heaving and pitching motions. This effect is exhibited in the result with the pitching amplitude as little as only a small fraction ( $\sim 0 \cdot 1$ or less) of the heaving motion, provided the phase difference is correctly observed.

The foregoing exposition of the optimum solution leaves very little clue as to whether there also exists an optimum range of $\sigma$ (aside from the understanding 
that $\sigma$ should be sufficiently greater than $\sigma_{c}$ ) for practical operations. A possible source for such crucial information lies in the knowledge of the thrust contribution coming from the leading-edge suction, as was pointed out by Lighthill (1970), for the following reason. Although this suction force has been simplified to appear mathematically as a singular force acting on a pointed leading edge, it can be realized physically only when the thin section's leading edge is sufficiently rounded. The magnitude of this suction is therefore of utmost importance to its being realizable or not in practice.

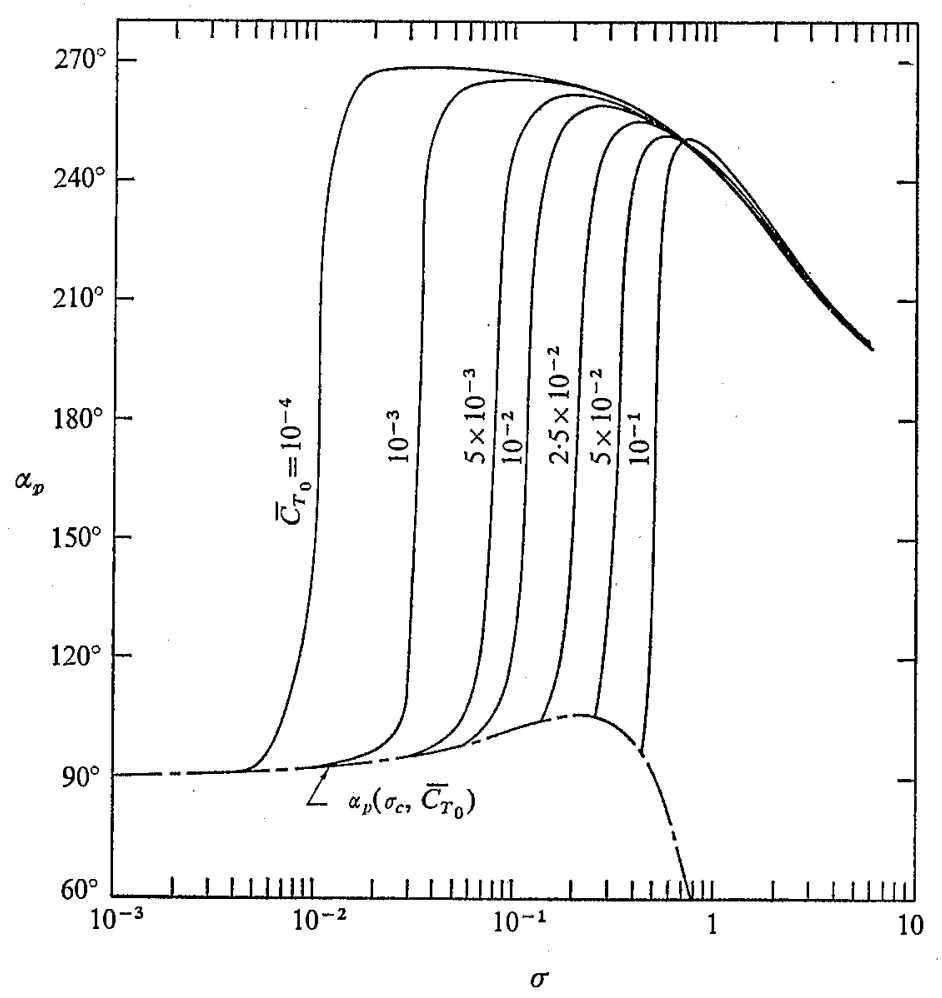

Fraure 7. The phase advance angle $\alpha_{p}\left(\sigma, \bar{C}_{T, 0}\right)$ of the pitching mode.

The leading-edge suction is given by (see part 1 , equations (43), (62))

$$
T_{S}=\frac{1}{8} \pi \rho\left(a_{0}+a_{0}^{*}\right)^{2}, \quad a_{0}=\left(b_{0}+b_{1}\right) \Theta(\sigma)-b_{1} .
$$

Its time average in harmonic motions is clearly

$$
\bar{T}_{S}=\frac{1}{4} \pi \rho a_{0} a_{0}^{*},
$$

which can be expressed in terms of $\xi_{0}, \xi_{1}, \xi_{2}$ for the motion given by (17) by a straightforward substitution upon using $(9 a),(28)$, yielding for the ratio of the mean suction thrust coefficient $C_{S} \equiv \bar{T}_{S} /\left(\frac{1}{4} \pi \rho U^{2} l\right)$, to the prescribed total thrust coefficient $C_{T}=C_{T, 0}$ as

$$
\begin{aligned}
C_{S} / C_{T}=\left(\bar{C}_{T, 0}\right)^{-1}\{[ & \left.(\sigma \mathscr{G}-2 \mathscr{F}) \frac{\xi_{1}}{\xi_{0}}-P_{22} \frac{\xi_{2}}{\xi_{0}}+\sigma \mathscr{G}\right]^{2} \\
& \left.+\left[(\sigma \mathscr{G}-2 \mathscr{F}) \frac{\xi_{2}}{\xi_{0}}+P_{22} \frac{\xi_{1}}{\xi_{0}}-\sigma \mathscr{F}\right]^{2}\right\} .
\end{aligned}
$$


For the optimum movement, $\xi_{1} / \xi_{0}$ and $\xi_{2} / \xi_{0}$ in (55) assume the values given by (51). The final result is plotted in figure 8 for various proportional-loading parameter $\bar{C}_{T, 0}$. It is of great interest to note that the ratio $C_{S} / C_{T}$ has a minimum at $\sigma=\sigma_{m}\left(\vec{C}_{T^{\prime}, 0}\right)$ say, and is relatively small in a short stretch of $\sigma$ about $\sigma_{m}$. Outside of this range, $C_{S} / C_{T}$ increases rapidly beyond 1 and becomes so large (the complementary thrust delivered by the plate surface is then negative) as to be certainly difficult to realize in practice without leading-edge stalling. It is also noteworthy that $\sigma=\sigma_{m}$ is very near the corresponding maximum of the $\alpha_{p}(\sigma)$ curve about which $\alpha_{p}$ varies relatively slowly with $\sigma$. It is thus convincing that the optimum range of operating $\sigma$ in practice should be somewhere very near $\sigma_{m}$, most likely to be a little greater than $\sigma_{m}$ before $C_{S} / C_{T}$ rises sharply so that a slightly improved efficiency can be achieved without risking to cause stall.

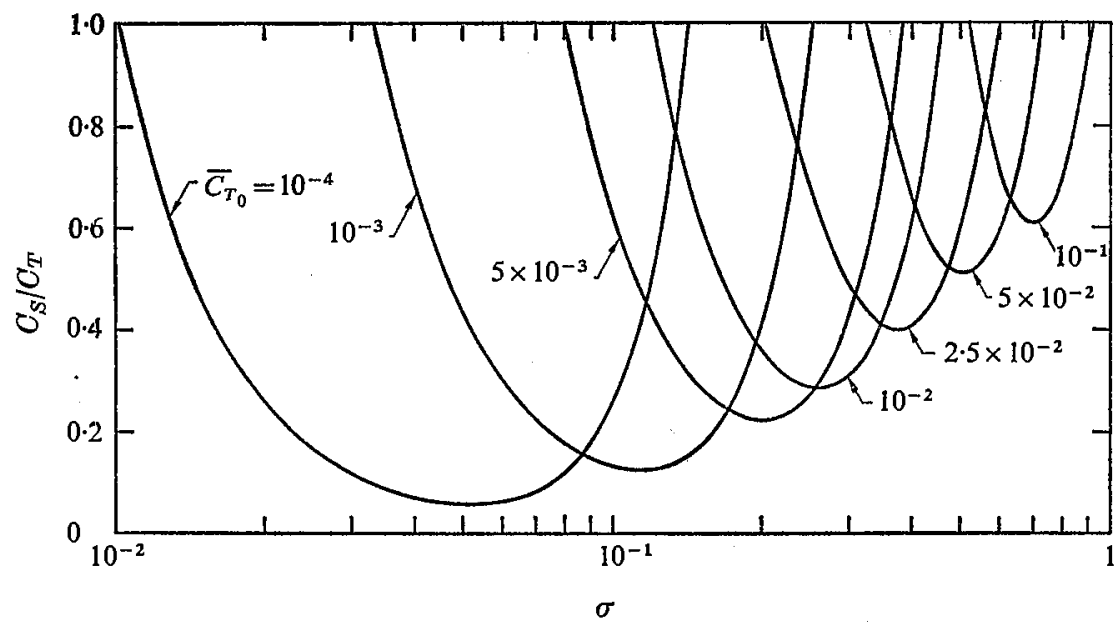

FIGURE 8. The ratio of the thrust coefficient $C_{S}$ due to leading-edge suction to the total thrust coefficient $C_{T}$.

The general problem of optimum movement of a rigid plate was investigated by Lighthill (1970); this study was known to the author when the present paper was written. It is thought to be of interest to discuss these independently arrived-at conclusions. Lighthill takes the section's lateral displacement in the form

$$
y=[h-i \alpha(x-b)] \exp (i \omega t) \quad(-l<x<l),
$$

where $h$ and $\alpha$ are real numbers signifying the amplitude of the heaving and pitching motions respectively, and $x=b, y=0$ is the axis of pitch. Clearly, Lighthill's adopting a fixed phase difference of $90^{\circ}$, while generalizing the axis of pitch, is equivalent to adopting a general phase difference between heaving and pitching-about-mid-chord-axis. In fact, this equivalence is completed by introducing a reference phase $\gamma$ to (17), and recovering the half-chord length $l$,

$$
y=\left[\frac{1}{2} l \xi_{0}+\left(\xi_{1}+i \xi_{2}\right) x\right] \exp [i(\omega t+\gamma)] \quad(-l<x<l) .
$$


Then the above two expressions of $y$ are equivalent if

$$
\begin{aligned}
b / l & =-\frac{1}{2} \xi_{0} \xi_{1}\left(\xi_{1}^{2}+\xi_{2}^{2}\right)^{-1}=-\frac{1}{2} Z_{p}^{-1} \cos \alpha_{p}, \\
h / l & =-\frac{1}{2} \xi_{0} \xi_{2}\left(\xi_{1}^{2}+\xi_{2}^{2}\right)^{-\frac{1}{2}}=-\frac{1}{2} \xi_{0} \sin \alpha_{p}, \\
\alpha & =\left(\xi_{1}^{2}+\xi_{2}^{2}\right)^{\frac{1}{2}}=\xi_{0} Z_{p} .
\end{aligned}
$$

As a useful measure of the relative magnitudes of pitching and heaving, Lighthill $(1969,1970)$ introduced a 'proportional-feathering parameter', $\theta=U \alpha / \omega h$, which is found to be indicative of thrust and efficiency considerations. Physically, this parameter provides a measure of the deviation of the plate slope from the tangent to the path traversed in the space by the axis of pitch. Since this path is sinusoidal, the largest value $\alpha$ can assume for positive thrust is the maximum slope of the path, $\alpha=k h, k$ being the wave-number, which gives

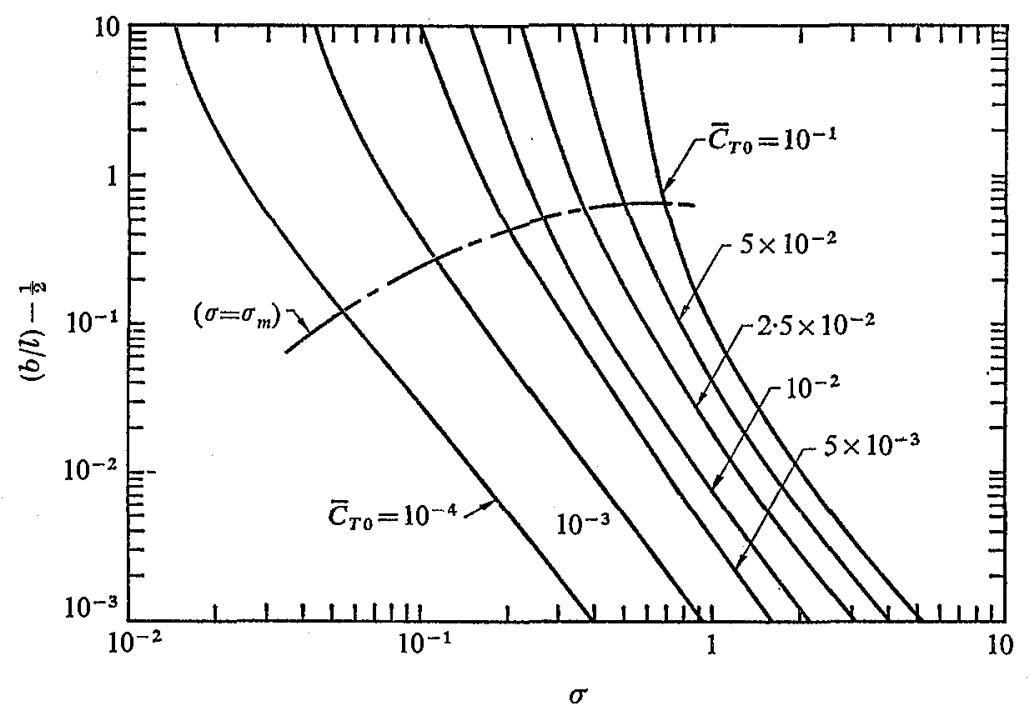

FreURe 9. The optimum location of pitching axis $x=b$ when the heaving is taken to lead the pitching by $90^{\circ}$ in phase. The dotted chain line denotes $\sigma=\sigma_{m}$ along which the leading-edge suction is minimum.

$\theta=U k / \omega=U / c$, where $c$ is the wave velocity relative to the plate. Thus, $\theta$ is usually less than 1 , and $\theta=1$ corresponds to geometrically accurate feathering of the fin. In terms of the present notation, $\theta$ can also be written as

$$
\theta \equiv \frac{U \alpha}{\omega h}=-\frac{2}{\sigma}\left(\xi_{1}^{2}+\xi_{2}^{2}\right) / \xi_{0} \xi_{2}=-\frac{2}{\sigma} Z_{p} \csc \alpha_{p}
$$

The advantage of Lighthill's form (56) first appears in the result that the wasted energy in the wake has a sharp minimum when $b=\frac{1}{2} l$, or when the pitching axis is at the $\frac{3}{4}$-chord point, whereas the rate of working increases somewhat for axis positions $b$ distal to that. Consequently, an optimum from thrust considerations as well as from efficiency considerations lies somewhere between $b=\frac{1}{2} l$ and $b=l$ (i.e. for the pitching axis to lie between the $\frac{3}{4}$-chord point and the trailing edge).

The present optimization, however, is held under an extra isoperimetric 
condition (10) for fixed thrust. The corresponding results of the optimum values of $b / l$ and $\theta$ for given $\bar{C}_{T, 0}$, by using (51) in (57) and (60), are plotted in figures 9,10 , in which the dotted chain lines correspond to $\sigma=\sigma_{m}$, along which the leading-edge suction is the smallest possible. Along this line, the pitch-axis $b$ increases from $\frac{1}{2} l$ to $\frac{3}{2} l$ whereas the feathering parameter $\theta$ falls off from 1 to 0 as $\bar{C}_{T, 0}$ increases. These general features are in qualitative agreement with the

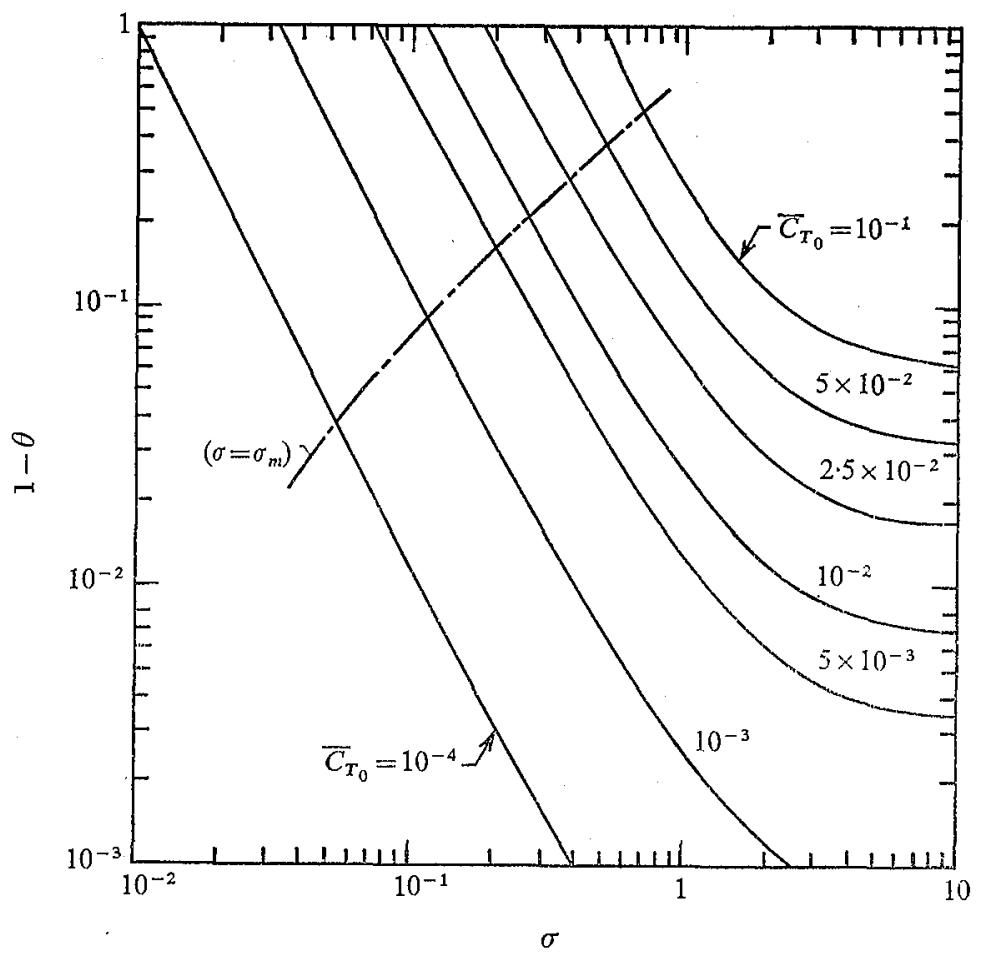

FIGURE 10. Variation of the feathering parameter $\theta$ (defined by (60)) with the reduced frequency $\sigma$. The dotted chain line denotes $\sigma=\sigma_{m}$ along which the leading-edge suction is minimum.

predictions of Lighthill (1970). As a further remark here, we note that the point $b / l=\frac{1}{2}, \theta=1$ is readily seen, by $(29),(57)$ and $(60)$, to be equivalent to $\xi_{1}=\hat{\xi}_{1}, \xi_{2}=\xi_{2}$, of which the significance has already been discussed.

For further comparison of the theory with experiments we proceed to discuss the following specific numerical example.

\section{Movements of porpoise tail}

Lang \& Daybell (1963) reported a series of experiments dealing with the swimming performance of a porpoise (of genus Lagenorhyncus obliquidens, or the Pacific Whitesided Dolphin) who was trained to swim and glide along an almost straight course in a long towing tank. This was perhaps one of the very few exhaustive and carefully conducted tests of a live cetacean under a wellcontrolled condition. The following data, which are thought to be useful for a qualitative comparison, are cited from Lang \& Daybell (1963). 
The porpoise, $6.7 \mathrm{ft}$. long, had a total body surface of $S=16.8 \mathrm{ft}^{2}$, including the tail surface area $0.527 \mathrm{ft}^{2}$. The tail, nearly triangular but slightly crescent in shape, had a span of $1.69 \mathrm{ft}$. and a maximum central chord of $0.625 \mathrm{ft}$., corresponding to an aspect-ratio of $5 \cdot 4$. The total drag $D$ was estimated for full laminar, full turbulent, and $40 \%$ laminar flows at various porpoise speeds based on known test data for rigid, smooth ellipsoidal bodies. A particular run selected for the present study was a stretch at porpoise speed $U=17 \mathrm{ft} . / \mathrm{sec}$, even though a slight acceleration was also recorded. The drag coefficient $C_{D}=D /\left(\frac{1}{2} \rho U^{2} S\right)$ equal to 0.0027 based on $40 \%$ laminar flow at this speed (or the laminar region Reynolds number of $4 \cdot 2 \times 10^{6}$ ) seems to agree fairly well with the observed drag derived from the deceleration measurement during glide runs, though the data of the latter kind have a considerable scatter. This value of $C_{D}$ will be taken as a representative case for comparison. The amplitude of tail stroke, as measured from this particular run (run no. 15-22, see figure 11) was about $10 \mathrm{in}$., or $0.83 \mathrm{ft}$. The tail angles of attack, measured relative to the undulating path traversed by the tail base, are also shown in figure 11 as given by Lang, who remarked on the considerable difficulty of determining the accuracy of the data. As thought to be most likely, the large size of the tail, its great vertical movement, and its noticeable changes in angle of attack would all suggest that a major part (perhaps more than $50 \%$ ) of the total thrust was produced by the tail alone, leaving the remainder to be generated by the body movement.

Since the aspect-ratio of the tail is sufficiently large to justify the strip theory, we shall adopt this approach, using the local two-dimensional characteristics for each strip. Just as a qualitative estimate we shall further simplify the strip integration by using its algebraic mean, though this will over-estimate the thrust and efficiency. Assuming the total drag $D$ is balanced by the tail thrust during the cruising period, we find

$$
C_{T, 0}=D /\left(\frac{1}{4} \pi \rho U^{2} \frac{1}{2} S_{\text {ta11 }}\right)=(4 / \pi)\left(S / S_{\text {tai1 }}\right) C_{D}=40 \cdot 5 C_{D}=0 \cdot 11,
$$

where $C_{T}$ stands for the local two-dimensional characteristic. The amplitude of tail stroke of $0.83 \mathrm{ft}$., when referred to an effective mean half-chord of the tail, $l=0.2 \mathrm{ft}$. (which is taken to be slightly on the larger side in order to account for the missing part of the body contribution to the thrust) gives in dimensionless form:

and hence

$$
\begin{gathered}
\xi_{0} / 2=(0 \cdot 83) /(0 \cdot 2), \quad \text { or } \quad \xi_{0}=8 \cdot 3, \\
\bar{C}_{T, 0}=C_{T, 0} / \xi_{0}^{2}=1 \cdot 6 \times 10^{-3} .
\end{gathered}
$$

The corresponding value of $\sigma_{c}$, by (46), is about $\sigma_{c} \simeq 0.01$. The wavelength of the track of the tail base is estimated from figure 11 to be about $\lambda=5.5 \mathrm{ft}$., corresponding to the reduced frequency of the tail motion,

$$
\sigma=\frac{\omega l}{U}=\frac{2 \pi l}{\lambda} \sim 0 \cdot 4 \pi / 5 \cdot 5=0 \cdot 228,
$$

which is very large compared with $\sigma_{c}$, but is quite close to $\sigma_{m}$ for this $\bar{C}_{T, 0}$.

Now, suppose this tail movement was performed at the optimum efficiency. Then, the efficiency, the amplitude ratio $Z_{p}$, and the phase advance $\alpha_{p}$ of the 
pitching mode of the tail are found from figures 6,7 at the above $\vec{C}_{T, 0}$ and $\sigma$ to be

$$
\eta=0.99, \quad Z_{p}=\left(\xi_{1}^{2}+\xi_{2}^{2}\right)^{\frac{1}{2}} / \xi_{0}=0 \cdot 104, \quad \alpha_{p}=\tan ^{-1} \xi_{2} / \xi_{1}=263^{\circ} .
$$

The tail motion, upon taking the real part, is

$$
h=\frac{1}{2} \xi_{0} \cos \omega t+\left(\xi_{1} \cos \omega t-\xi_{2} \sin \omega t\right) x \quad(-1<x<1),
$$

where $h$ and $x$ are both referred to the mean half-chord $l=0.2 \mathrm{ft}$. The tail angles of attack relative to the free stream is

$$
-\partial h / \partial x=-\xi_{0} Z_{p} \cos \left(\omega t+\alpha_{p}\right)=-0.862 \cos \left(\omega t+263^{\circ}\right)
$$

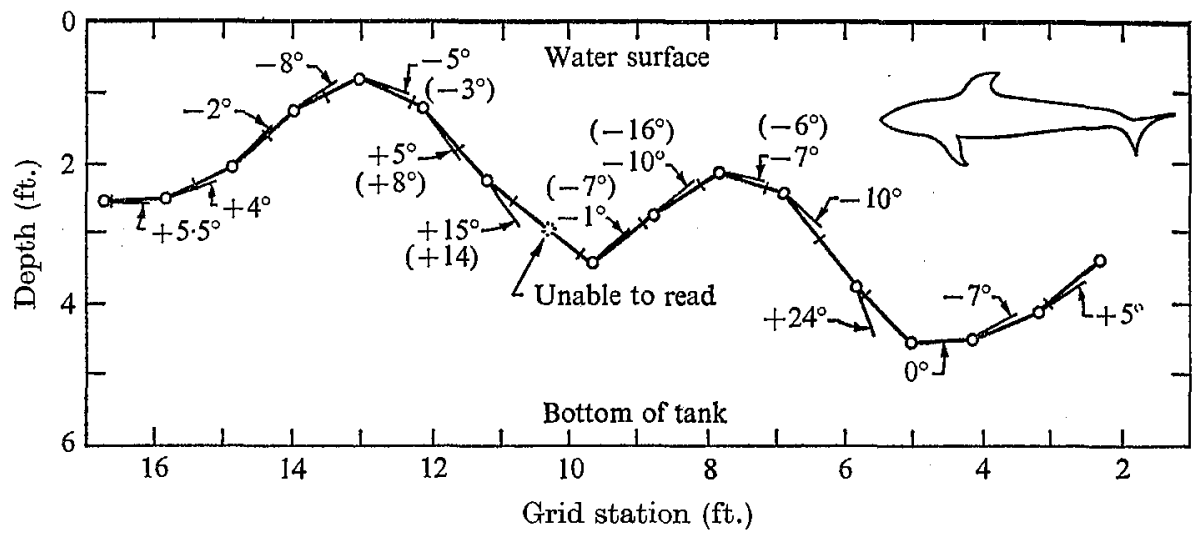

Fraure 11. Tail movements of a porpoise in cruising. The angles with arrows are the incidence angles of the tail relative to the path of tail-base measured by Lang \& Daybell (1963); the angles in parentheses are the present theoretical prediction at the corresponding positions. (Experimental data $\rightarrow-$, courtesy of Dr T. G. Lang.)

The slope of the path traversed by the tail base was observed, quite approximately, as $d y / d x=0.55 \sin \omega t$. Hence, the tail angles of attack relative to the path traversed by the tail base is

$$
\alpha_{\text {tall }}=d y / d x-\partial h / \partial x \simeq 0.55 \sin \omega t-0.862 \sin \left(\omega t-7^{\circ}\right)
$$

The angles $\alpha_{\text {ta11 }}$ predicted by (65) are shown in figure 11 within parentheses directly below the experimental data of Lang. This comparison, however, should be properly qualified, since the application of the two-dimensional theory tends to overestimate the efficiency, determination of the effective mean chord is crude, and the accuracy of the measured $\alpha_{\text {tall }}$ was claimed to be somewhat uncertain. These rather obscure circumstances notwithstanding, it is still of significance to observe that the general trend of the predicted $\alpha_{\text {tall }}$ is in fair accordance with the experimental measurements.

In terms of Lighthill's form (56) of the lateral motion, the location of the pitch-axis corresponding to the $Z_{p}$ and $\alpha_{p}$ given by (64) assumes the value, by $(57)$,

$$
b / l=\frac{1}{2}\left(\sin 7^{\circ}\right) /(0 \cdot 104)=0 \cdot 585,
$$

and the corresponding feathering parameter is, by $(60)$,

$$
\theta=(0 \cdot 208 / 0 \cdot 228)\left(\sec 7^{\circ}\right)=0.92 \text {. }
$$


The above value of $b / l$ locates the pitch axis at $\frac{1}{2}(1+0.585)=0.793$-chord point from the leading edge, which is well in the favourable range predicted by Lighthill (1970, see particularly his figure 4). The value of feathering parameter $\theta=0.92$ is somewhat higher than the range 0.6 to 0.8 discussed by Lighthill, but it is in the right direction for higher efficiencies. Finally, an interpolation check with figures 8-1.0 shows that the observed reduced frequency $\sigma=0.228$ is somewhat greater than the $\sigma_{m}$ (which is about $0 \cdot 14$ for the $\bar{C}_{T, 0}$ at hand), the leading-edge suction at this $\sigma$ is, nevertheless, still reasonably small,

$$
C_{S} / C_{T} \simeq 0 \cdot 4
$$

In conclusion, the following comments are perhaps in order about the main features of the tail movement. (i) The estimated reduced frequency $\sigma=0 \cdot 228$ is large compared with $\sigma_{c}=0 \cdot 01$, but lies well in the range in which the leadingedge suction is not large. (ii) The loading parameter $\bar{C}_{T, 0}\left(=1.6 \times 10^{-3}\right.$ as estimated) turns out to be very small, mainly owing to the large amplitude of heaving. (iii) The phase difference $\alpha_{p}=263^{\circ}$ between the pitching (about the mid-chord) and heaving modes falls in the range of $\sigma$ where $\alpha_{p}$ is nearly stationary, and is 'safely' away from the region of rapid changes of $\alpha_{p}$. (iv) With pitching kept only at a rather small amplitude $\left(Z_{p}=0.11\right.$ in this case) but with the correct phase $\alpha_{p}$, impressively high efficiency $(\eta \sim 0.99)$ can be achieved. (v) When the heaving is forcibly made to lead the pitching by $90^{\circ}$ in phase, the pitch axis is at about 0.8 -chord point, and the feathering $(\theta=0.92)$ is nearly accurate. It seems quite conclusive that (i) is the primary condition for selecting the frequency $\sigma$ in practice.

\section{Movements of bird's wing in flapping flight}

The present two-dimensional theory can also be used to discuss qualitatively the optimal movement of a bird's wing in flapping flight as most species of migrating birds have wings of high aspect-ratio, and there must be a considerable saving of energy with optimum wing movement. We shall again adopt the strip theory to give a first-order estimate, leaving the effect of finite span as a further refinement. A somewhat superficial difference between fish propulsion and bird flight arises from the need in the latter case of adding to the oscillatory motion the constant angle of attack required for supporting the body weight in air. But this steady component can be accounted for separately; it does not correlate with the oscillatory component in the balance of mean energy.

Take the $z$-axis to lie along the mean position of the wing span, with the wing stretched from $z=-b$ to $b$. The wing motion is assumed to have primarily a heaving and a pitching mode, so that, for a wing strip at station $z$, the up-anddown flapping displacement in the $y$-direction can be written

$$
h=\left\{\frac{1}{2} \xi_{0}(z)+\left[\xi_{1}(z)+j \xi_{2}(z)\right] x\right\} \exp (j \omega t) \quad((x, z) \in S),
$$

where $S$ denotes the plane form of the wing, and the amplitude functions $\xi_{n}$ 's generally depend on $z$, they being real and even functions of $z$ for symmetrical 
motions. Ordinarily, bending of the wing is relatively small except possibly near the tip. To fix our ideas, we assume

$$
\xi_{0}(z)=c_{0}\left(z-z_{c}\right) \quad(0<z<b),
$$

so that $\left(c_{0} z_{c}\right)$ gives the amplitude of the vertical displacement of body centroid, which is probably quite small in general. The $z$-dependence of $\xi_{1}$ and $\xi_{2}$ can then be discussed qualitatively based on the argument of optimum efficiency.

Suppose for simplicity that the chord is nearly constant along the span, except in the vicinity of the wing tip, so that the reduced frequency $\sigma$ referred to the local half-chord is almost uniform. To simplify the picture, we further assume that the spanwise distribution of the thrust coefficient $C_{T}$ is approximately constant, and fixed as required for overcoming the viscous drag. According to the present optimum solution, high efficiencies very close to unity can be achieved if $\sigma$ is sufficiently greater than $\sigma_{c}$, and if the local $\bar{C}_{T, 0}=C_{T, 0} / \xi_{0}^{2}$ is sufficiently small, a condition which can be satisfied by making the amplitude $\xi_{0}$ of flapping large. This high efficiency $\eta$ implies that $C_{P}$ will be nearly equal to $C_{T}$ (since $1-C_{T} / C_{P}=1-\eta \ll 1$ ), and hence also will be almost uniformly distributed along the span. However, since the flapping amplitude $\xi_{0}(z)$ grows monotonically outwards from $z=z_{c}$, we have

$$
\partial \bar{C}_{T, 0} / \partial z=-\left(2 C_{T, 0} / \xi_{0}^{3}\right)\left(d \xi_{0} / d z\right)<0 \quad\left(z>z_{c}\right)
$$

so that $\bar{C}_{T, 0}$ decreases rapidly towards the wing tip. Figure 6 then indicates that the amplitude ratio $Z_{p}=\left(\xi_{1}^{2}+\xi_{2}^{2}\right)^{\frac{1}{2}} / \xi_{0}$ should increase slightly with $z$, implying that $\left(\xi_{1}^{2}+\xi_{2}^{2}\right)^{\frac{1}{2}}$ should increase at least at the same rate as $\xi_{0}$ towards the wing tip. Furthermore, figure 7 indicates that the phase advance angle $\alpha_{p}$ of pitching should also increase with decreasing $\bar{C}_{T, 0}$ as $z$ moves towards the wing tip. In this range of $\sigma, \alpha_{p}$ is somewhat smaller than $270^{\circ}$. The general picture is then roughly as follows: As the wing flaps up and down, the pitching amplitude increases with the distance outward from the body, reaching a nearly horizontal position at the top and bottom of each stroke. Such a wing movement, according to this simple strip-theory argument, is the most efficient, and leaves behind the least possible vorticity in over.coming a given frictional drag. This crude picture may be further refined by employing a more accurate lifting-line or lifting-surface theory, and by including physiological considerations about limitations of physical structure, muscular power, metabolic rate and other factors. Such a broad study is, however, out of the scope of present considerations.

\section{The general optimum shape problem}

As soon as the shape function $h(x, t)$ of a flexible plate is allowed to have a higher number of possible modes, with more Fourier coefficients $\beta_{0}, \beta_{1}, \beta_{2}, \ldots$, $\beta_{N}(2<N \leqslant \infty)$ admitted to $h$ (see (2)), the optimum shape problem immediately becomes more involved. To begin with, we note that the degree of complexity of the analysis depends somewhat on the primary, but crucial, step of choosing the independent variable between $h$ and $V$. If $h$ is taken as the independent 
function, having the Fourier expansion (2), the Fourier coefficients of $V$ (see (4)) can be expressed in terms of the $\beta_{n}$ 's as

$$
b_{n} / U=j \sigma \beta_{n}+2 \sum_{s=0}^{\infty}(2 s+n+1) \beta_{2 s+n+1} \quad(n=0,1,2, \ldots) .
$$

The analysis subsequent to this approach can be developed along a line very much similar to the previous case of two-term expansion discussed in $\S 3$, the major step being again the reduction of the singular quadratic form $C_{E}$ to a non-singular one of a lower order. It turns out that the first non-singular reduced quadratic form of $C_{E}$ is always of order 2 regardless of the value $N(>2)$ to begin with. This is not surprising, since $C_{E}$ depends on only the first two Fourier coefficients $b_{0}, b_{1}$ of $V$. This property of $C_{E}$ also explains the advantages of taking $V$ as the independent function.

It is convenient first to decompose $V$ as

$$
\begin{gathered}
V(x, t) \exp (-j \omega t) / U=\left(c_{1}+j c_{2}\right) \cos \theta+V_{\perp}(x) \quad(x=\cos \theta), \\
V_{\perp}(x)=c_{0}\left(\frac{1}{2}-\cos \theta\right)+\sum_{n=2}^{\infty}\left(c_{2 n-1}+j c_{2 n}\right) \cos n \theta
\end{gathered}
$$

in which the $c_{n}$ 's are all real, and the coefficient of the constant term is taken to be purely real as a reference phase. By comparison with (4),

$$
b_{0} / U=c_{0}, \quad b_{1} / U=\left(c_{1}-c_{0}\right)+j c_{2}, \quad b_{n} / U=c_{2 n-1}+j c_{2 n} \quad(n=2,3, \ldots),
$$

in which the time factors $\exp (j \omega t)$ of the $b_{n}$ 's are omitted as understood. The above representation of $V$ is complete, and is so decomposed that $V_{\perp}(x)$ is orthogonal to $(1+x)$, i.e. by $(12)$,

$$
\left(V_{\perp}, \mathrm{I}+\cos \theta\right)=0
$$

whence

$$
(V, 1+\cos \theta)=b_{0}+b_{1}=U \exp (j \omega t)\left(c_{1}+j c_{2}\right) \text {. }
$$

The plate movement $h(x, t)$ corresponding to the above $V$ can be determined by integration of (3), giving

$$
\begin{aligned}
h(x, t)=\exp (-j \sigma x)\left\{\int_{0}^{\infty} \exp (j \sigma \xi) \frac{V(\xi, t)}{U} d \xi+\frac{1}{2}\left(\xi_{5}+j \xi_{6}\right) \exp (j \omega t)\right\} & \\
& (-1<x<1),
\end{aligned}
$$

where $\left(\xi_{5}+j \xi_{6}\right)$ is a constant of integration, which becomes known once $h(0, t)$ is prescribed. Upon substituting (71) in (75),

$$
\begin{array}{cr}
h(x, t) \exp (-j \omega t)=\frac{c_{1}+j c_{2}}{\sigma^{2}}(\mathrm{I}-j \sigma x-\exp (-j \sigma x))+h_{\perp}(x)+\frac{1}{2}\left(\xi_{5}+j \xi_{6}\right) \exp (-j \sigma x), \\
h_{\perp}(x)=\exp (-j \sigma x) \int_{0}^{x} \exp (j \sigma \xi) V_{\perp}(\xi) d \xi .
\end{array}
$$

Again we note that $h$ can admit a progressing wave $\exp [j(\omega t-\sigma x)]$ without affecting $V$. 
The Fourier coefficients $\beta_{n}$ 's of $h$ can be derived from (76) by making use of the Fourier-Bessel expansion (Watson 1944, p. 22)

$$
\exp ( \pm j \sigma \cos \theta)=J_{0}(\sigma)+2 \sum_{n=1}^{\infty}( \pm j)^{n} J_{n}(\sigma) \cos n \theta
$$

The first two coefficients are determined as

$$
\begin{aligned}
\beta_{0} \exp (-j \omega t) & =2\left(c_{1}+j c_{2}\right) \frac{1-J_{0}(\sigma)}{\sigma^{2}}+\left(\xi_{1}+j \xi_{2}\right)+\left(\xi_{5}+j \xi_{6}\right) J_{0}(\sigma) \\
\beta_{1} \exp (-j \omega t) & =\frac{c_{1}+j c_{2}}{j \sigma}\left[1-\frac{2}{\sigma} J_{1}(\sigma)\right]+\left(\xi_{3}+j \xi_{4}\right)-j\left(\xi_{5}+j \xi_{6}\right) J_{1}(\sigma), \\
\xi_{1}+j \xi_{2} & =\frac{2}{\pi} \int_{0}^{\pi} h_{\perp}(x) d \theta, \quad \xi_{3}+j \xi_{4}=\frac{2}{\pi} \int_{0}^{\pi} h_{\perp}(x) \cos \theta d \theta
\end{aligned}
$$

Upon substituting (74) and (78) into (6)-(7), we obtain

$$
\begin{aligned}
& C_{E}=B(\sigma)\left(c_{1}^{2}+c_{2}^{2}\right), \\
& C_{P}=\widetilde{P}_{22}\left(c_{1}^{2}+c_{2}^{2}\right)+\sigma \widetilde{A}_{1} c_{1}+\sigma \widetilde{A}_{2} c_{2},
\end{aligned}
$$

where

$$
\begin{gathered}
\tilde{P}_{22}=(1-\mathscr{F})\left[1-\frac{2}{\sigma} J_{1}(\sigma)\right]+\frac{2}{\sigma} \mathscr{G}\left[1-J_{0}(\sigma)\right], \\
\tilde{A}_{1}+j \tilde{A}_{2}=\left(\xi_{1}+j \xi_{2}\right)(\mathscr{G}+j \mathscr{F})-\left(\xi_{3}+j \xi_{4}\right)[\mathscr{G}-j(1-\mathscr{F})] \\
+\left(\xi_{5}+j \xi_{6}\right)\left[(\mathscr{G}+j \mathscr{F}) J_{0}(\sigma)+(1-\mathscr{F}+j \mathscr{G}) J_{1}(\sigma)\right] .
\end{gathered}
$$

The present result (79)-(80) is now seen to be analogous to the previous case of rigid plate, (35)-(37). Proceeding in a similar way, we extremize $C_{E}$, with again $C_{T}=C_{T, 0}$ fixed (see (10)), by varying first $c_{1}$ and $c_{2}$. The variational solution, containing $\sigma, C_{T, 0}$ as well as $\left(\xi_{1}, \ldots, \xi_{6}\right)$ as a family of parameters, is of the form,

$$
c_{1}=\lambda \tilde{A_{1}}, \quad c_{2}=\lambda \tilde{A_{2}},
$$

$\lambda$ being a Lagrange multiplier. By substituting (81) in (79)-(80)

$$
C_{E}=B(\lambda \tilde{A})^{2}, \quad C_{P}=\left(\tilde{P}_{22} \lambda^{2}+\sigma \lambda\right) \tilde{A}^{2}, \quad \tilde{A}^{2}=\left|\tilde{A}_{1}+j \tilde{A}_{2}\right|^{2} .
$$

Application of condition (10) now yields

$$
\begin{gathered}
\widetilde{T}_{22} \lambda^{2}+\sigma \lambda=\widetilde{C}_{T, 0}, \\
\text { where } \quad \widetilde{T}_{22}=\widetilde{P}_{22}-B, \quad \tilde{C}_{T, 0}=C_{T, 0} /(\widetilde{A})^{2} .
\end{gathered}
$$

In this case, $\lambda$ again has two solutions:

$$
\left.\begin{array}{l}
\lambda_{1} \\
\lambda_{2}
\end{array}\right\}=\frac{\sigma}{2 \widetilde{T}_{22}}\left\{-1 \pm(1+\tilde{\Lambda})^{\frac{1}{2}}\right\}, \quad \tilde{\Lambda} \equiv \frac{4}{\sigma^{2}} \widetilde{T}_{22} \tilde{C}_{r, 0} .
$$

In comparing this result with (42) of the rigid plate case, we note that the general feature of $\widetilde{T}_{22}(\sigma)$ is quite similar to $T_{22}$, namely, $\widetilde{T}_{22}$ vanishes at $\sigma=0$ and $\sigma=\tilde{\sigma}_{0}=2 \cdot 51$, and

$$
\widetilde{T}_{22}(\sigma) \gtrless 0 \quad \text { according as } \quad \sigma \gtrless \tilde{\sigma}_{0}=2 \cdot 51 \text {. }
$$

The derivative $d \widetilde{T}_{22} / d \sigma$ is found to be appreciably smaller than $d T_{22} / d \sigma$, as is shown in figure 1. From this property it follows that, if the parameter $\tilde{C}_{T, 0}$ of 
this solution assumes the same value as $\bar{C}_{T, 0}$ in the rigid plate case, the rate of increase $d \tilde{\Lambda} / d \sigma$ is slower than $d \Lambda / d \sigma$, and hence the critical reduced frequency $\tilde{\sigma}_{c}<\sigma_{c}$, where $\tilde{\Lambda}\left(\tilde{\sigma}_{c}, \tilde{C}_{T, 0}\right)=-1$, and $\sigma_{c}$ is given by (43). Consequently, $\lambda_{1}$ and $\lambda_{2}$ will be real if $\tilde{\Lambda} \geqslant-1$, or

$$
\sigma \geqslant \tilde{\sigma}_{c}\left(\tilde{C}_{T, 0}\right) \text { where } \tilde{\Lambda}\left(\tilde{\sigma}_{c}, \tilde{C}_{T, 0}\right)=-1 .
$$

Within this range of $\sigma, \lambda_{1}$ corresponds to the maximum efficiency,

$$
\eta_{\max }=\frac{C_{T, 0}}{C_{P}}=1-\frac{C_{R}}{C_{P}}=1-\frac{B \lambda_{1}}{\tilde{P}_{22} \lambda_{1}+\sigma} \quad\left(\sigma \geqslant \tilde{\sigma}_{c}\right),
$$

whereas $\lambda_{2}$ yields the minimum efficiency

$$
\eta_{\min }=C_{T, 0} / C_{P, \max }=1-\frac{B \lambda_{2}}{\tilde{P}_{22} \lambda_{2}+\sigma} \quad\left(\sigma \geqslant \tilde{\sigma}_{c}\right) .
$$

The present optimum solution, being not yet subjected to the recoil conditions, contains the parameters $\sigma, C_{T, 0}, \xi_{1}, \xi_{2}, \ldots \xi_{6}$. When these eight parameters are prescribed, $\lambda_{1}$ is given by (84), $c_{1}$ and $c_{2}$ by (81), and the optimum profile $h$ is furnished by (76), except the component $h_{\perp}(x)$ is determinate only up to the first two Fourier coefficients (see (78c)). To this end, we note that the Fourier coefficients of $h_{\perp}(x)$ higher than the second have no influence upon the optimum efficiency. Furthermore, it is of significance to observe that the parameters $\xi_{1}, \xi_{2}, \ldots \xi_{6}$ appear in the solution of $\eta_{\max }$ only through the quantity $\tilde{A}^{2}$, which is a quadratic form of $\xi_{1}, \xi_{2}, \ldots \xi_{6}$ with frequency-dependent coefficients. Consequently, every point $\left(\xi_{1}, \xi_{2}, \ldots \xi_{6}\right)$ on the quadrics $\widetilde{A}^{2}=$ const. will yield the same $\eta_{\max }$. This result shows that the optimum solution, as presently posed, can be determined only to a certain degree, but not to the extent to provide a unique $h(x, t)$. The reason for this, as mentioned earlier, is because there appears in this variational problem only a few scalar products involving $h$ and $V$.

Judging from the known properties of the rigid plate solution, which are quite similar to the present general case, it can be inferred that for fixed $\sigma$ and $C_{T, 0}, \eta_{\max }$ will increase with decreasing $\tilde{\Lambda}$, or with increasing $\widetilde{A}^{2}$ (see (84)). Furthermore, $\eta_{\max }$ very close to unity can be attained when $C_{T, 0} /(\tilde{A})^{2} \ll 1$.

The actual numerical work can be facilitated by first expressing the quadratic form $\widetilde{A}^{2}$ in the canonical form. A possible choice of the new variables is

$$
\zeta_{1}=\xi_{1}-\xi_{1}\left(\xi_{3}, \ldots \xi_{6}\right), \quad \zeta_{2}=\xi_{2}-\xi_{2}\left(\xi_{3}, \ldots \xi_{6}\right)
$$

where $\xi_{1}$ and $\hat{\xi}_{2}$ are the values of $\xi_{1}$ and $\xi_{2}$, respectively, which will make $\tilde{A}_{1}+j \widetilde{A}_{2}$ and hence also $\widetilde{A}^{2}$ vanish for arbitrary $\left(\xi_{8} \ldots \xi_{6}\right)$. Then $(80 c)$ becomes

hence

$$
\begin{gathered}
\tilde{A}_{1}+j \tilde{A}_{2}=(\mathscr{G}+j \mathscr{F})\left(\zeta_{1}+j \zeta_{2}\right), \\
\tilde{A}^{2}=\left(\mathscr{F}^{2}+\mathscr{G}^{2}\right)\left(\zeta_{1}^{2}+\zeta_{2}^{2}\right),
\end{gathered}
$$

this being in the canonical form. Clearly, $\eta_{\max }$ depends on two parameters $\left[\sigma, C_{T, 0} /\left(\zeta_{1}^{2}+\zeta_{2}^{2}\right)\right], c_{1}$ and $c_{2}$ depend on four parameters $\left(\sigma, C_{T, 0}, \zeta_{1}, \zeta_{2}\right)$, whilst $\beta_{0}$ and $\beta_{1}$ depend on $\left(\sigma, C_{T, 0}, \zeta_{1}, \zeta_{2}, \xi_{3}, \xi_{4}, \ldots \xi_{6}\right)$. The above canonical form is not unique. The expression for $\widetilde{A}_{1}+j \widetilde{A}_{2}$ in $(80 c)$ indicates several other combinations of new variables. For instance, as another set, one may take

$$
\zeta_{3}=\xi_{3}-\hat{\xi}_{3}\left(\xi_{1}, \xi_{2}, \xi_{5}, \xi_{6}\right), \quad \zeta_{4}=\xi_{4}-\hat{\xi}_{4}\left(\xi_{1}, \xi_{2}, \xi_{5}, \xi_{6}\right)
$$


where $\hat{\xi}_{3}$ and $\hat{\xi}_{4}$ are such that $\tilde{A}_{1}+j \tilde{A}_{2}$ vanishes at $\xi_{3}=\hat{\xi}_{3}, \xi_{4}=\hat{\xi}_{4}$. Then

$$
\tilde{A}_{1}+j \tilde{A}_{2}=-[\mathscr{G}-j(1-\mathscr{F})]\left(\zeta_{3}+j \zeta_{4}\right),
$$

and the corresponding form of $\widetilde{A}^{2}$ is again canonical.

For each of these canonical forms of $\tilde{A}^{2}$, the calculation of the optimum $\eta_{\max }$ will be entirely parallel to the special case of the rigid plate, although there remain additional free parameters in the determination of the optimum shape. It may be expected that due to the additional degrees of freedom admitted to $h(x, t)$ for this general case, $\eta_{\max }$ will be further improved from the rigid plate value at fixed $\sigma$ and $C_{T, 0} / \xi_{0}^{2}, \xi_{0}$ being the heaving amplitude.

Finally, suppose the body recoil conditions (15)-(16) are also to be satisfied, then these two conditions will give four scalar equations relating four more parameters of $V$, say $c_{3}, c_{4}, c_{5}, c_{6}$, to the remaining unknown coefficients. This shows that $h(x, t)$ as given by (76) can be determined to a higher degree, the lack of complete determinateness of the optimum shape remains, nevertheless, an intrinsic feature of the problem.

I am deeply indebted to Professor M. J. Lighthill for interesting and stimulating discussions, and particularly for calling my attention to the significance of leading-edge suction in this problem. My remarks in this paper would have been less complete had I not had the privilege of knowing his important contribution (1970) prior to its publication. I am also grateful to Dr T. G. Lang for discussion on his experiments, and to Professors C. R. De Prima and Duen-pao Wang for their interest in the general problem presented in $\S 6$. Assistance provided by Dr Arthur Whitney and Mr Allen Chwang in numerical studies is greatly appreciated. This work was partially sponsored by the National Science Foundation, under Grant GK 10216, and by the Office of Naval Research, under Contract N00014-67-A0094-0012.

\section{REFERENCES}

Lang T. G. \& DaybetL, D. A. 1963 NA VWEPS Rept. 8060; NOTS Tech. Publ. 3063.

LigसтнгLL, M. J. 1969 Ann. Rev. Fluid Mech. 1, 413.

LigHTHILL, M. J. 1970 J. Fluid Mech. 44, 265.

WANG, P. K. C. 1966 IEEE Trans. Automatic Control, AC 11, 645.

Watson, G. N. 1944 A Treatise on the Theory of Bessel Functions (2nd edn.). Cambridge University Press.

Wu, T. Y. 1971 J. Fluid Mech. 46, 337. 